\title{
Metformin intervention against ovarian toxicity during chemotherapy for early breast cancer: study protocol for a randomized double-blind placebo- controlled trial
}

\section{Jinjin Zhang}

Huazhong University of Science and Technology Tongji Medical College

\section{Xiangyi Ma}

Tongji Hospital of Tongji Medical College of Huazhong University of Science and Technology Ya Li

Tongji Hospital of Tongji Medical College of Huazhong University of Science and Technology Ronghua Liu

Tongji Hospital of Tongji Medical College of Huazhong University of Science and Technology Yan Li

Tongji Hospital of Tongji Medical College of Huazhong University of Science and Technology

Panshi Zhang

Tongji Hospital of Tongji Medical College of Huazhong University of Science and Technology

\section{Wu Ren}

Tongji Hospital of Tongji Medical College of Huazhong University of Science and Technology

\section{Pengfei Cui}

Tongji Hospital of Tongji Medical College of Huazhong University of Science and Technology

\section{Bo Wang}

Tongji Hospital of Tongji Medical College of Huazhong University of Science and Technology

\section{Minli Zhang}

Tongji Hospital of Tongji Medical College of Huazhong University of Science and Technology

\section{Yan Jin}

Tongji Hospital of Tongji Medical College of Huazhong University of Science and Technology

\section{Xingrui Li}

Huazhong University of Science and Technology

Shixuan Wang ( $\sim$ sxwang@tjh.tjmu.edu.cn )

Huazhong University of Science and Technology Tongji Medical College

\section{Study protocol}


Keywords: Metformin, Breast cancer, Chemotherapy, Ovarian toxicity

Posted Date: January 29th, 2020

DOI: https://doi.org/10.21203/rs.2.22188/v1

License: (c) (i) This work is licensed under a Creative Commons Attribution 4.0 International License. Read Full License 


\section{Abstract}

Background With the significant improvement of the cure rate and survival rate of cancer patients, the survivors need to face quality of life problems, such as significant decline in reproductive system development, ovarian reserves and function, and even fertility loss and early menopause. These problems are often highly associated with chemotherapy-induced ovarian damage in the course of cancer treatment. However, there are no ideal treatment strategies at present. In our attempt to develop reagents and approaches for delaying ovarian aging and protecting chemotherapy-induced ovarian injury, we recently found that metformin may be the most potential drug to protect female malignant tumor patients from chemotherapy-induced ovarian injury. The optional trial is aimed to test whether administration of metformin during chemotherapy could protect normal ovarian function of early breast cancer patients.

Methods This study is prospective, randomized, double-blind and placebo-controlled. Female early breast cancer patients ( $\mathrm{N}=314)$, were randomly assigned to two groups (placebo, metformin $2000 \mathrm{mg}$ ). Metformin was administered during and after chemotherapy for patients with stage I-Illa breast cancer. The primary outcome was the menstruation recovery rate 12 months after chemotherapy, defined as recovery of menstruation twice in a row within 1 year. Patients were followed up for 5 years to observe long-term ovarian function and prognosis of tumor, such as overall survival (OS), objective response rate (ORR), disease-free survival (DFS). Quality of life and safety will also be assessed.

Discussion Our research will provide new treatment strategy of fertility protection, and clinical treatment guidance for cancer patients.

\section{Contributions To The Literature}

This protocol demonstrates how to utilize implementation science methodologies to conduct RCT trials against ovarian toxicity during chemotherapy for early breast cancer patients.

This study will provide a potential alternative agent for fertility protection of tumor patients, and provide guidance for the implementation of clinical programs.

This study may provide a useful strategy for the improvement of life quality and prognosis of tumor such as OS, ORR, DFS of cancer patients.

This project will be of great practical significance and long-term strategic significance to alleviate the huge economic pressure caused by tumor rejuvenation.

\section{Background}

Globally, the incidence of cancer still shows the trend of rapid growth, particularly in developing countries [1]. In China, breast cancer ranks first in the incidence of female population ${ }^{[2]}$. Over the past two decades, due to the rapid development of medical technology, the survival rate of cancer patients has been significantly improved. The most common cancer in women aged 15-59 is breast cancer, with an incidence of $24.2 \%$ and a 5 -year survival rate of more than $70 \%{ }^{[3]}$. However, with the significant 
improvement of the cure rate and survival rate of cancer patients, the survivors need to face quality of life problems, such as maintaining normal female reproductive function and fertility.

Cancer survivors have a significant decline in reproductive system development, ovarian reserves and function, and even fertility loss and early menopause, which are the life problems female survivors have to suffer of ${ }^{[4]}$. These problems are often highly associated with chemotherapy-induced ovarian damage in the course of cancer treatment. Gonads are very sensitive to chemotherapeutic drugs, especially when being long-term exposure to cyclophosphamide that causes serious damage to the development and function of the female reproductive system ${ }^{[5]}$. This type of chemotherapeutic drugs is widely used in the treatment of breast cancer. Studies have shown that the ovarian reserve of 15-39 years old cancer survivors undergoing chemotherapy is significantly lower than that of unexposed women in the same age group, and their ovarian reserve and function are close to those of normal women in peri-menopausal period ${ }^{[6]}$. Early menopause can also increase the risk of menopause-related diseases, such as cardiovascular disease, osteoporosis, depression and anxiety, seriously affecting the quality of life of patients. Therefore, the chemotherapy-induced ovarian injury in female cancer patients remains to be a key issue, and the protection of gonad development and fertility in cancer patients of all ages is urgently needed.

Chemotherapy-induced ovarian injury has been payed the close attention by multidisciplinary oncologists. A number of studies to prevent the injury have been carried out. The currently available protective measures include embryo cryopreservation, mature oocyte cryopreservation, ovarian tissue cryopreservation and reimplantation, and gonadotropin releasing hormone analogues ${ }^{[7]}$. However, these protective measures have not been ideally fruitful with a relatively low success rate, inaccurate efficacy, and narrow scope of application, while mostly invasive operation may delay chemotherapy and lead to secondary cancer. As such, their current clinical application is still limited. Therefore, there is an urgent need to find a new approach to reduce the ovarian damage caused by chemotherapeutic drugs without affecting the effect of chemotherapy, and to provide effective protection for the development of reproductive system and fertility in female patients with breast cancer.

In our attempt to develop reagents and approaches for delaying ovarian aging and protecting chemotherapy-induced ovarian injury, we recently found that metformin may be the most potential drug to protect female malignant tumor patients from chemotherapy-induced ovarian injury. As a first-line drug for type 2 diabetes (T2D), metformin has been used in clinical practice for 60 years with no toxicity to patients. Although it is an "old drug", the actual and potential uses of metformin go far beyond its prescription for for T2D. Metformin has been shown to play an important role in cancer therapy [8], delaying aging ${ }^{[9]}$, reversing pulmonary fibrosis ${ }^{[10]}$, protecting the cardiovascular system ${ }^{[11]}$ and regulating intestinal flora ${ }^{[12]}$. There are a number of relevant basic studies and clinical trials on the use of metformin in various diseases. Also, the Food and Drug Administration (FDA) recently approved the clinical trial of metformin for an anti-aging study, the first anti-aging drug study in human beings. Several studies have shown that the combination of metformin and chemotherapy does not reduce the efficacy 
of chemotherapy ${ }^{[13,14]}$. Therefore, metformin represents as a promising candidate drug for protecting female patients from chemotherapy-induced ovarian injury over traditional reproductive protection measures.

In the female reproductive system, metformin has been shown to reduce insulin resistance, restore ovulation function and improve hyperandrogenemia in patients with polycystic ovary syndrome ${ }^{[15]}$. A recent study showed that metformin could combat amenorrhea caused by psychotic drugs, $66.7 \%$ of the subjects in the metformin intervention group recovered menstruation, while only $4.8 \%$ of the placebo group ${ }^{[16]}$. In line with this finding, we recently found that metformin significantly improves ovarian reserve, endocrine and reproductive function in middle-aged mice ${ }^{[17]}$.

Based on these findings, we propose to carry out a clinical study of metformin administration against chemotherapy-induced ovarian damage for female patients with breast cancer. Completing this study will provide useful information and a potential alternative agent for the improvement of life quality and fertility protection of tumor patients, and provide guidance for the implementation of clinical programs.

\section{Methods/design}

\section{Study design}

This study is designed as a randomized, double-blind, placebo-controlled trial with allocation of 1:1. The trial is registered at Chinese Clinical Trial Registry on 31 May 2019 (ChiCTR1900023487) and enrolment started in November 2019. The recruitment is expected to be completed in November 2022. See Additional file for the CONSORT checklist.

\section{Patients}

Three hundred and fourteen patients fulfilling the eligibility criteria below will be included.

\section{Inclusion criteria}

- Female adults aged $18-45$ years old;

- I-Illa ER (-) PR (-) HER-2 (-) or HER-2 (+) breast cancer;

- Those who will receive adjuvant / neoadjuvant chemotherapy;

- Normal ovarian function before chemotherapy (regular menstruation 2 months before chemotherapy);

- Those who signed the informed consent form.

\section{Exclusion criteria}


- Those who have previously received chemotherapy and pelvic radiotherapy;

- Endocrine diseases affecting ovarian function (such as polycystic ovary syndrome (PCOS), hyperprolactinemia, hyperandrogenemia, abnormal adrenal function, etc.);

- Complicated with cardiovascular, liver, kidney, infectious diseases, hematopoietic system and other serious diseases, lactic acid poisoning history or high-risk factors;

- Distant metastasis of breast cancer;

- Pregnant or lactating women;

- Having a history of diabetes and currently taking any hypoglycemic drug;

- Those who are known to be allergic to metformin and its analogues;

- Patients who are participating in or have participated in other clinical trials in the past 3 months;

- Those who took contraceptive or hormone drugs orally during the trial;

- Those who were identified by the researchers as unfit to participate in the study;

\section{Sample size calculation}

The sample size calculation is based on the following assumptions. Ovarian reserve / function recovery rate of chemotherapy-induced amenorrhea in patients with breast cancer is $45 \%$ [18]. The study will be powered to detect a difference in change of 20 points between the Metformin and Control groups in the primary outcome (Menstruation recovery rate) from baseline to 12 months follow-up. To provide $90 \%$ power using a one-sided test at the confidence level of 0.025 , with $1: 1$ randomization and assuming a drop-out rate of $20 \%$, each group will take 157 cases, and a total of 314 subjects will be recruited.

\section{Recruitment procedure}

The overall trial flow is outlined in Figure 1. Patients meeting the inclusion criteria will be invited to participate in the RCT study. Doctors, nurses and clinical research coordinators (CRC) involved in recruitment have been trained and instructed in the recruitment procedure in order to maximise the recruitment rate. Oral and written information about the study will be provided by the examining doctors at the department of breast surgery in Tongji hospital. Patients are recommended to take at least 24 hours to consider and discuss participation with a relative or a lay representative before deciding on participation in the study.

Figure1. The overall trial flow of the study.

\section{Randomization procedure and concealment of allocation}

In this study, stratified block randomization will be used. Before randomization, the subjects will be stratified according to the age of breast cancer (18-29y; 30-34y; 35-45y). The subjects will be randomized 
to the metformin group and the placebo group at a rate of 1:1. The patients will be given a random number according to the chronological order of entry, and the researchers will give the subjects corresponding treatment according to the random grouping. Random coding is released as a separate part to reduce experimental bias and information disclosure. A sealed random form will remain in the center where the project applicant is located.

\section{Blinding}

This is a double-blind study. Randomization schedule will be created by an independent randomization statistician. All participants and study team (including investigators, outcome assessors, and data analysis statisticians) will be blinded to group allocation throughout the study. In order to ensure the successful implementation of the blinding method, the products in both groups are similar in smell and dosage form, and packed in identical packages. Researchers, patients, and caregivers are unaware of the treatment allocation until completion of the study.

\section{Intervention}

\section{Standard Chemotherapy regimen}

For ER(-) PR(-) HER-2(-) breast cancer patients, EC-T or AC-T chemotherapy regimen is optional. And for HER-2(+) breast cancer patients, EC-HखAC-H or TCb-H regimen can be chosen.

\section{Metformin or placebo taken}

All the patients will be diagnosed with breast cancer before entering the group, and the stage will be determined after examination by 2 professors. If the other inclusion conditions were met, they could be randomly divided into metformin group and placebo group. After entering the group, the eligible patients will be treated according to the treatment plan, and during the period of chemotherapy, the patients in the trial group will be given metformin adjuvant therapy, and the patients in the control group will be treated with placebo.

In the first week of chemotherapy, one tablet $(500 \mathrm{mg} /)$ per day will be taken with lunch on the first week, and two tablets $(1000 \mathrm{mg})$ per day on the second week, one tablet at lunch and one tablet at dinner. From the third week to one month after the end of chemotherapy, four tablets per day, two tablets for lunch and two tablets for dinner, taken with or immediately after meal.

\section{Data collection procedure}

Data will be collected at baseline, before chemotherapy (adjuvant / neoadjuvant chemotherapy), every 3 months during the chemotherapy and 3, 6, 9 and 12 months after chemotherapy, then lasted for 5 years 
every half a year. Follow-up will be assessed at the hospitals by project workers specifically trained in the test protocol. Please refer to Table 1 for an overview of collection of the different outcomes.

\section{Outcomes}

\section{Baseline characteristics}

Patients' characteristics such as height, body weight index (BMI), hormones levels, and menstruation status will be collected before chemotherapy.

\section{Primary outcome}

The primary outcome is menstruation recovery rate, which is defined as recovery of menstruation twice in a row within 1 year after chemotherapy.

\section{Secondary outcomes}

Other ovarian reserve/function markers: Ovarian recovery will be assessed by other markers such as serum levels of sex hormone such as anti-Müllerian hormone (AMH), inhibin $B$, estrogen, progestogen, testosterone, prolactin (PRL), follicle stimulating hormone (FSH) and luteinizing hormone (LH).

Pregnancy rate in 5 years: Patients' reproduction function will be assessed by the proportion of successful conception and parturition of patients who tried to conceive after treatment.

Prognosis of tumor: The prognosis of tumor will be assessed by OS, ORR, DFS.

Quality of life: Patients' quality of life will be assessed by depression anxiety scale and climacteric syndrome kupperman scale. Patients' liver and kidney function, blood routine, blood biochemistry, coagulation function and imaging examination will be evaluated during the chemotherapy and follow-up period.

\section{Adverse Events}

The researcher should carefully observe any adverse events that occurred during the clinical study, and require the subjects to truthfully reflect the changes of their condition after treatment and avoid induced questions. While observing the curative effect, pay attention to the adverse reactions or unexpected side effects (including symptoms, signs and laboratory examination). Regardless whether the adverse events are related to the experimental drugs or not, they should be recorded in detail in the CRF table, including the occurrence time, symptoms, signs, degree, duration, laboratory examination indicators, methods of treatment, process, results, follow-up time, etc., and the situation of combined use of drugs should be recorded in detail in order to analyze the correlation between the adverse events and the experimental drugs, and the record should be signed and dated.

When adverse reactions are found, the researchers can take necessary treatment measures according to the condition, such as adjusting the dose, temporarily interrupting the use of drugs, and decide whether to 
terminate the trial. When a serious adverse event occurs, the unit responsible for the experimental study must immediately take necessary measures to protect the safety of the subjects.

\section{Data management}

Patient characteristics will be recorded in CRFs, which will be stored in the researcher's work office, and codes and initials will be used instead of the participants' information to protect the participants' privacy. Quality of CRF completion will be monitored by the specified researcher. Researchers must ensure that the CRF is finished completely and accurately. The data of only one clinical study subject is recorded in each case report table. All incorrect data or text cannot be altered, but must be marked with a single line, then re-fill in the correct data or text on the side, and sign the name and revision date of the researcher.

All the original RCT data will be entered into the Yi du scientific research collaboration platform. The relevant users will be trained and the system will be tested before it is officially launched to ensure that the system meets the trial requirements. Only relevant personnel will receive the account number and password once the system is officially launched. The clinical supervisor will monitor the work of the clinical trial center at least once a month.

\section{Monitoring}

To improve the quality of this study, the whole process will be conducted under the supervision of a qualified clinical trial expert and a clinical research supervisor. The clinical research supervisor has responsibility to verify that the clinical trial is conducted in strict accordance with the clinical research plan, and solve the problems that arise in any clinical research with the researchers. If any details of this protocol are changed, the decisions will be recorded by the medical ethics committee, and then be announced to the persons conducting the trial. The PI will take full responsibility and will make the final decision. The supervising nurse has the responsibility to compare the clinical research records with the subjects' original medical records in order to ensure the authenticity of the clinical data.

All drugs and materials used in all clinical studies must be based on quality control. Key researchers and project applicants or relevant medical management agencies have the right to review clinical research in order to ensure the authenticity of clinical research records and comply with the requirements of clinical research programs. Subjects in clinical studies will be informed that there is a review process for clinical studies, but patients' privacy and data will be strictly protected.

\section{Statistical analysis}

Statistical analysis plans are drawn up by biostatisticians and leading researchers according to the research program to improve the documentation before blindness and data locking. Statistical analysis software applies SAS 9.1 or later. Significant level $=0.05$ (bilateral). 
The distribution of all subjects who signed the ICF will be provided. The number of subjects who were screened, randomly grouped, completed and discontinued during the study period, as well as the reasons for discontinuation of the study after all random grouping, were summarized according to the treatment group. The distribution and reasons for the discontinuation of the study by subject will also be provided.

- Demographic and baseline characteristics

Demographic and baseline characteristics will be summarized by treatment group using appropriate descriptive statistics. The mean, median, standard deviation, minimum and maximum values were used to summarize the continuous variables according to the treatment of the study, and the differences will be analyzed by t-test or Wilcoxon rank sum test. The frequency count and percentage will be used to summarize the variables according to the treatment of the study, and the chi-square test will be used to analyze the difference.

- Analysis of curative effect

- Main outcome measures, ovarian reserve / function recovery rate, chi-square test or Fisher's exact test will be used to analyze whether metformin group is superior to placebo group. If there are unbalanced covariables between the test group and the control group, the $\mathrm{CMH}$ chi-square or logistic regression model is used to correct the covariables.

- Other ovarian reserve/function markers: ovarian recovery will be assessed by other markers such as serum levels of sex hormone such as $\mathrm{AMH}$, inhibin $\mathrm{B}$, estrogen, progestogen, testosterone, prolactin $(\mathrm{PRL})$, follicle stimulating hormone ( $\mathrm{FSH}$ ) and luteinizing hormone (LH). Chi-square test or Fisher's exact test will be used to analyze whether the metformin group is superior to the placebo group.

- Pregnancy: the proportion of successful conception and fertility of patients who tried to conceive after treatment (except for children). Chi-square test or Fisher's exact test will be used to analyze whether the metformin group is superior to the placebo group. If there are unbalanced covariables between the test group and the control group, the $\mathrm{CMH}$ chi-square or logistic regression model is used to correct the covariables.

- Tumor prognosis: from the date of completion of randomization, overall survival (OS) and diseasefree survival (DFS) were described by Kaplan Meier method, and compared by log-rank test. The hazard ratios in the experiment group compared with control group will be estimated by Cox regression model. ORRs of the two groups were compared using chi-square or Fisher's exact test, and the odds ratios will be estimated by logistic regression.

- For the quality of life of patients, for the scale data, t-test or wilcoxon rank sum test will be used to analyze the difference.

- Safety Analysis.

To summarize the frequency and percentage of adverse events and severe adverse events in metformin group and placebo group. Adverse events and their most serious response levels will be summarized in 
accordance with NCI CTCAE version 4.0 standards, and adverse events will be summarized based on the severity of the event and its relationship to the drug studied.

The descriptive summary of laboratory test values is mainly aimed at outliers. Laboratory inspection anomalies will also be summarized according to the most severe level of NCI CTCAE version 4.0.

\section{Dissemination and protocol amendments}

The primary RCT results will be submitted for publication to an international, peer-reviewed journal, regardless of whether the results are positive, negative or inconclusive in relation to the study hypothesis. Authorship eligibility will be based on the recommendations from the International Committee of Medical Journal Editors (ICMJE). Any important protocol amendments will be reported to the Regional Committees on Health Research Ethics for Southern Denmark, registered at Chinese Clinical Trial Registry and communicated in the primary RCT report.

\section{Discussion}

Considering the difficulty in recruiting patients and referring to lots of previous studies, the dose of metformin is fixed. In spite of this limitation, the study will either provide new strategies for protection against ovarian toxicity during chemotherapy for early breast cancer, and provide high-quality evidence supporting the role of metformin in fertility preservation. We believe that the successful implementation of this project will provide valuable information and alternative prevention for improving quality life of female patients with breast cancer. Either way, the results will provide scientific support for doctors and patients when discussing the optimal treatment option for the individual patient. In the long run, the project will have important practical significance and long-term strategic significance to alleviate the huge economic pressure and social harmony and stability caused by the rejuvenation of tumor.

\section{Declarations}

\section{Ethics approval and consent to participate}

This study is approved by Medical Ethics Committee of Tongji Medical College, Huazhong University of Science and Technology. Detail has been removed from this case description/these case descriptions to ensure anonymity. The editors and reviewers have seen the detailed information available and are satisfied that the information backs up the case the authors are making.

\section{Consent for publication}

Not applicable. 


\section{Availability of data and materials}

The associated study protocol and data collection tools will be made available upon request from the corresponding author. Quantitative datasets are available from the corresponding author upon reasonable request after the completion of primary analyses and results dissemination. Qualitative study datasets will not be available, as they may include identifiable information that could comprise participant identity.

\section{Competing interests}

The authors affirm that they have no competing interests.

\section{Funding}

This research was supported by the grant from International S\&T Cooperation Program of China (No.2013DFA31400), and grants from the pilot project of Tongji Hospital, Tongji Medical College, Huazhong University of Science and Technology.

\section{Authors' contributions}

Jinjin Zhang and Shixuan Wang conceived the trial and led the development of all procedures including intervention design, data management and statistical analyses and drafted the first version of the manuscript. Yan Li, Ronghua Liu, Xiangyi Ma, and Xingrui Li contributed to conceptualization, recruitment and data collection. Wu Ren, Pengfei Cui, Bo Wang and Minli Zhang contributed to cancer patient recruitment and data collection. Shixuan Wang and Xingrui Li contributed to conceptualization, methodology, and supervision. All authors contributed to the review and editing of the manuscript, read and approved the final version.

\section{Acknowledgements}

We thank the staff of the department of thyroid and breast surgery, and the staff of the scientific research office, and the statisticians for their work on this project.

\section{References}

1. Freddie Bray J, Ferlay, Soerjomataram. I. Global cancer statistics 2018: GLOBOCAN estimates of incidence and mortality worldwide for 36 cancers in 185 countries. CA Cancer J Clin. 2018;68(6):394424. 
The latest National. Cancer report in 2018, China national cancer center.

3.

Morgan S, et al., How do chemotherapeutic agents damage the ovary? Human Reproduction Update, 2012. 18(5): p. 525-535.

4.

Benedict C, Shuk E, Ford JS. Fertility Issues in Adolescent and Young Adult Cancer Survivors. J Adolesc Young Adult Oncol. 2016;5(1):48-57.

5 .

Bhakta $\mathrm{N}$, et al. The cumulative burden of surviving childhood cancer: an initial report from the St Jude Lifetime Cohort Study (SJLIFE). The Lancet. 2017;390(10112):2569-82.

6.

Gracia CR, et al., Impact of cancer therapies on ovarian reserve. Fertil Steril, 2012. 97(1): p. 134 - 40 e1. 7.

Ahmad SS, et al. Anticancer chemotherapy in teenagers and young adults: managing long term side effects. BMJ. 2016;354:i4567.

8.

al WD. H.D.e., Glucose-regulated phosphorylation of TET2 by AMPK reveals a pathway linking diabetes to cancer. nature. 2018;559(7715):637-41.

9.

Cabreiro F. A.C.e.a., Metformin retards aging in C. elegans by altering microbial folate and methionine metabolism. cell. 2013;153(1):228-39.

10.

Rangarajan S. B.N., et al, Metformin reverses established lung fibrosis in a bleomycin model. Nat Med. 2018;24(8):1121-7.

11.

Soberanes S. M.A., et al, Metformin Targets Mitochondrial Electron Transport to Reduce Air-PollutionInduced Thrombosis. Cell Metab. 2018;p. S1550-4131(18):30586-2.

12.

Bauer PV. D.F., et al, Metformin Alters Upper Small Intestinal Microbiota that Impact a Glucose-SGLT1Sensing Glucoregulatory Pathway. Cell Metab. 2018;27(1):101-17.

13.

Hee Jeong Kim1, H.K., Jong Won Lee1, Hwa Jung Kim3, Sae Byul Lee1, Hee Sung Park1, Guiyun Sohn1, Yura Lee1, Beom Seok Koh1, Jong Han Yu1, Byung Ho Son1 and Sei Hyun Ahn1*, Metformin increases survival in hormone receptor-positive, HER2-positive breast cancer patients with diabetes. Breast Cancer Research, 2015.

14.

Tan BX, et al. Prognostic Influence of Metformin as First-Line Chemotherapy for Advanced Nonsmall Cell Lung Cancer in Patients With Type 2 Diabetes. Cancer. 2011;117(22):5103-11.

15.

Z

Page 13/16 
$X Z$, et al., Polycystic ovarian syndrome: Correlation between hyperandrogenism, insulin resistance and obesity. Clinica chimica acta; international journal of clinical chemistry, 2019.

16.

Wu RR. J.H., et al, Metformin for treatment of antipsychotic-induced amenorrhea and weight gain in women with first-episode schizophrenia: a double-blind, randomized, placebo-controlled study. Am J Psychiatry Am J Psychiatry. 2012;169(8):813-21.

17.

Qin X, Chen DD, Wu Q, Wu M, Wen T, Jin J, Zhang Y, Wang J. S, Metformin prevents murine ovarian aging. Aging. 2019 Jun;10(11):11.

18.

Zavos Senologic Hellenic Society A, Greece A. Risk of chemotherapy-induced amenorrhea in patients with breast cancer: a systematic review and meta-analysis. Acta Oncologica, 2016. 55(6).

\section{Tables}

Table 1₫An overview of collection of the different outcomes.

Note: M for months and CT for chemotherapy.

\section{Figures}




\begin{tabular}{|c|c|c|c|c|c|c|c|c|c|c|c|c|c|c|}
\hline & Baseline & $\begin{array}{c}3 \mathrm{M} \\
\text { initiated } \\
\mathrm{CT}\end{array}$ & $\begin{array}{l}3 \mathrm{M} \\
\text { after } \\
\mathrm{CT}\end{array}$ & $\begin{array}{c}6 \mathrm{M} \\
\text { after } \\
\mathrm{CT}\end{array}$ & $\begin{array}{l}9 \mathrm{M} \\
\text { after } \\
\mathrm{CT}\end{array}$ & $\begin{array}{c}12 \\
\mathrm{M} \\
\text { after } \\
\mathrm{CT}\end{array}$ & $\begin{array}{c}18 \mathrm{M} \\
\text { after } \\
\mathrm{CT}\end{array}$ & $\begin{array}{l}24 \mathrm{M} \\
\text { after } \\
\mathrm{CT}\end{array}$ & $\begin{array}{l}30 \mathrm{M} \\
\text { after } \\
\mathrm{CT}\end{array}$ & $\begin{array}{l}36 \mathrm{M} \\
\text { after } \\
\mathrm{CT}\end{array}$ & $\begin{array}{l}42 \mathrm{M} \\
\text { after } \\
\mathrm{CT}\end{array}$ & $\begin{array}{l}48 \mathrm{M} \\
\text { after } \\
\mathrm{CT}\end{array}$ & $\begin{array}{c}54 \\
\mathrm{M} \\
\text { after } \\
\mathrm{CT} \\
\end{array}$ & $\begin{array}{c}60 \\
\mathrm{M} \\
\text { after } \\
\mathrm{CT}\end{array}$ \\
\hline $\begin{array}{c}\text { Baseline } \\
\text { characteristics }\end{array}$ & & & & & & & & & & & & & & \\
\hline Age & $\sqrt{ }$ & & & & & & & & & & & & & \\
\hline BMI & $\sqrt{ }$ & & & & & & & & & & & & & \\
\hline Menstrual status & $\sqrt{ }$ & & & & & & & & & & & & & \\
\hline $\begin{array}{c}\text { Sex hormones } \\
\text { levels }\end{array}$ & $\sqrt{ }$ & & & & & & & & & & & & & \\
\hline $\mathrm{AMH}$ & $\sqrt{ }$ & & & & & & & & & & & & & \\
\hline Inhibin B & $\sqrt{ }$ & & & & & & & & & & & & & \\
\hline $\begin{array}{c}\text { Tumor } \\
\text { information }\end{array}$ & & & & & & & & & & & & & & \\
\hline Tumor size $\square \mathrm{T} \square$ & $\sqrt{ }$ & & & & & & & & & & & & & \\
\hline Axillary nodes $(\mathrm{N})$ & $\sqrt{ }$ & & & & & & & & & & & & & \\
\hline Tumor grade (G) & $\sqrt{ }$ & & & & & & & & & & & & & \\
\hline $\begin{array}{c}\text { Hormone } \\
\text { receptor status }\end{array}$ & $\sqrt{ }$ & & & & & & & & & & & & & \\
\hline $\begin{array}{c}\text { Chemotherapy } \\
\text { regimens }\end{array}$ & & & & & & & & & & & & & & \\
\hline $\begin{array}{c}\text { Timing of } \\
\text { chemotherapy } \\
\square \text { Adjuvant or } \\
\text { Neoadjuvant } \\
\end{array}$ & $\sqrt{ }$ & & & & & & & & & & & & & \\
\hline $\begin{array}{c}\text { Type of } \\
\text { chemotherapy }\end{array}$ & $\sqrt{ }$ & & & & & & & & & & & & & \\
\hline $\begin{array}{c}\text { Cumulative } \\
\text { cyclophosphamide } \\
\text { dose, median } \\
\text { (IQR), mg/m2 }\end{array}$ & $\sqrt{ }$ & & & & & & & & & & & & & \\
\hline $\begin{array}{c}\text { Duration of } \\
\text { chemotherapy, } \\
\text { median (IQR), wk }\end{array}$ & $\sqrt{ }$ & & & & & & & & & & & & & \\
\hline $\begin{array}{c}\text { Treatment } \\
\text { completed as } \\
\text { planned } \\
\text { Chemotherapy } \\
\end{array}$ & $\sqrt{ }$ & & & & & & & & & & & & & \\
\hline $\begin{array}{r}\text { Metformin or } \\
\text { placebo taken }\end{array}$ & $\sqrt{ }$ & & & & & & & & & & & & & \\
\hline $\begin{array}{l}\text { Adverse event } \\
\text { record }\end{array}$ & & $\sqrt{ }$ & $\sqrt{ }$ & $\sqrt{ }$ & $\sqrt{ }$ & $\sqrt{ }$ & $\sqrt{ }$ & $\sqrt{ }$ & $\sqrt{ }$ & $\sqrt{ }$ & $\sqrt{ }$ & $\sqrt{ }$ & $\sqrt{ }$ & $\sqrt{ }$ \\
\hline Tumor prognosis & & $\sqrt{ }$ & $\sqrt{ }$ & $\sqrt{ }$ & $\sqrt{ }$ & $\sqrt{ }$ & $\sqrt{ }$ & $\sqrt{ }$ & $\sqrt{ }$ & $\sqrt{ }$ & $\sqrt{ }$ & $\sqrt{ }$ & $\sqrt{ }$ & $\sqrt{ }$ \\
\hline $\begin{array}{c}\text { Routine } \\
\text { examination for } \\
\text { safetynBlood, } \\
\text { urine, ect. } \square\end{array}$ & $\sqrt{ }$ & $\sqrt{ }$ & $\sqrt{ }$ & $\sqrt{ }$ & $\sqrt{ }$ & $\sqrt{ }$ & $\sqrt{ }$ & $\sqrt{ }$ & $\sqrt{ }$ & $\sqrt{ }$ & $\sqrt{ }$ & $\sqrt{ }$ & $\sqrt{ }$ & $\sqrt{ }$ \\
\hline \multicolumn{15}{|l|}{$\begin{array}{c}\text { Ovarian function } \\
\text { recovery }\end{array}$} \\
\hline Menstrual status & $\sqrt{ }$ & $\sqrt{ }$ & $\sqrt{ }$ & $\sqrt{ }$ & $\sqrt{ }$ & $\sqrt{ }$ & $\sqrt{ }$ & $\sqrt{ }$ & $\sqrt{ }$ & $\sqrt{ }$ & $\sqrt{ }$ & $\sqrt{ }$ & $\sqrt{ }$ & $\sqrt{ }$ \\
\hline Hormones levels & $\sqrt{ }$ & $\sqrt{ }$ & $\sqrt{ }$ & $\sqrt{ }$ & $\sqrt{ }$ & $\sqrt{ }$ & $\sqrt{ }$ & $\sqrt{ }$ & $\sqrt{ }$ & $\sqrt{ }$ & $\sqrt{ }$ & $\sqrt{ }$ & $\sqrt{ }$ & $\sqrt{ }$ \\
\hline $\begin{array}{c}\text { Pregnancy } \\
\text { outcome }\end{array}$ & $\sqrt{ }$ & $\sqrt{ }$ & $\sqrt{ }$ & $\sqrt{ }$ & $\sqrt{ }$ & $\sqrt{ }$ & $\sqrt{ }$ & $\sqrt{ }$ & $\sqrt{ }$ & $\sqrt{ }$ & $\sqrt{ }$ & $\sqrt{ }$ & $\sqrt{ }$ & $\sqrt{ }$ \\
\hline $\begin{array}{c}\text { climacteric } \\
\text { syndrome } \\
\text { Kuppermann scale }\end{array}$ & $\sqrt{ }$ & $\sqrt{ }$ & $\sqrt{ }$ & $\sqrt{ }$ & $\sqrt{ }$ & $\sqrt{ }$ & $\sqrt{ }$ & $\sqrt{ }$ & $\sqrt{ }$ & $\sqrt{ }$ & $\sqrt{ }$ & $\sqrt{ }$ & $\sqrt{ }$ & $\sqrt{ }$ \\
\hline $\begin{array}{c}\text { Depression } \\
\text { anxiety scale }\end{array}$ & $\sqrt{ }$ & $\sqrt{ }$ & $\sqrt{ }$ & $\sqrt{ }$ & $\sqrt{ }$ & $\sqrt{ }$ & $\sqrt{ }$ & $\sqrt{ }$ & $\sqrt{ }$ & $\sqrt{ }$ & $\sqrt{ }$ & $\sqrt{ }$ & $\sqrt{ }$ & $\sqrt{ }$ \\
\hline
\end{tabular}




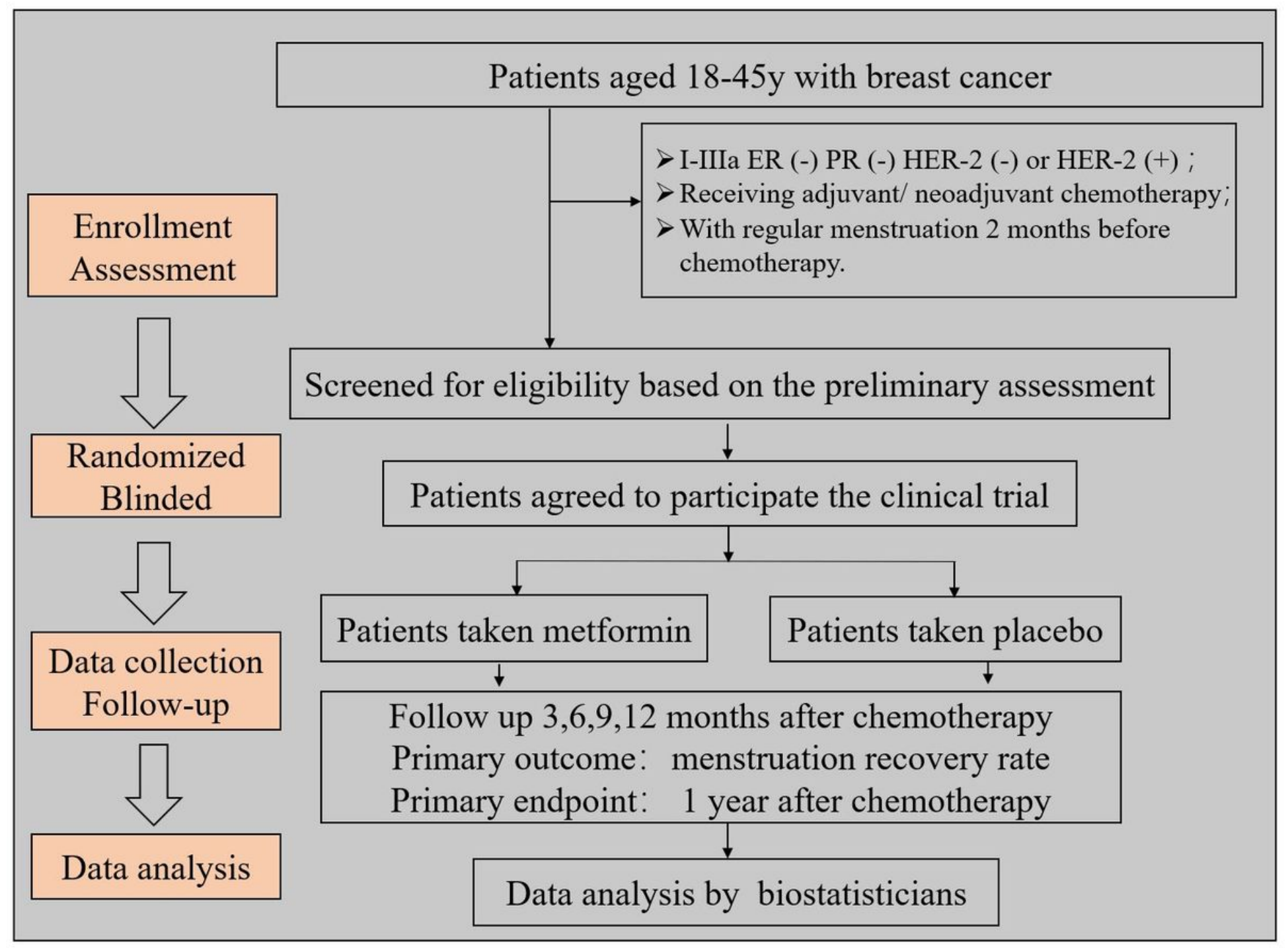

Figure 2

Trial flow

\section{Supplementary Files}

This is a list of supplementary files associated with this preprint. Click to download.

- CONSORTChecklist.doc

- CONSORTChecklist.doc 